\title{
A Simple Model for Magnetization Ratios in Doped Nanocrystals
}

\author{
Joshua Schrier and K. Birgitta Whaley \\ Department of Chemistry and Pitzer Center for Theoretical Chemistry, University of California, Berkeley 94720
}

\begin{abstract}
Recent experiments on Mn-doped ZnS nanocrystals have shown unusual magnetization properties. We describe a nearest-neighbor Heisenberg exchange model for calculating the magnetization ratios of these antiferromagnetically doped crystals, in which the dopant atoms are distributed inhomogeneously within the nanocrystal. This simple inhomogeneous doping model is capable of reproducing the experimental results, and suggests that interior dopant atoms are localized within the crystal.
\end{abstract}

\section{INTRODUCTION}

Transition metal doping is now possible for a variety of nanocrystalline semiconductor materials, e.g., CdS, 1.2 $\mathrm{CdSe}, \stackrel{3.4}{,}, \mathrm{ZnSe}, \stackrel{5}{5}$ and ZnS ${ }^{6.7}$ In bulk dilute magnetic semiconductors (DMS), Heisenberg coupling of magnetic dopants, such as Mn, occurs via anion-mediated superexchange $\stackrel{8.9}{=}$ but it is not completely understood how this is modulated by finite nanocrystal $(\mathrm{NC})$ size effects ${ }^{2}$

In a recent experiment, Tsuji et al. synthesized Mn-doped ZnS NCs and determined the ratio between the magnetization at a finite field and the expected magnetization for the given number of dopants per NC, defined as $M_{5 T} / M_{s a t} \underline{\underline{T}}^{7}$ The initially prepared NCs (designated "as prepared") had a 1.2\% Mn dopant concentration, an average of $4.3 \mathrm{Mn}$ dopants per $\mathrm{NC}$ (mostly on the surface), and $M_{5 T} / M_{s a t}=91 \%$. Washing the sample with acid removed the surface atoms, yielding a sample (designated "HCl-washed") with a $0.10 \% \mathrm{Mn}$ concentration, an average of 0.3-0.5 Mn dopants per NC (presumably on the interior), and $M_{5 T} / M_{\text {sat }}=77 \%$. The effect of antiferromagnetic coupling of the Mn ions is to reduce the magnetization ratio. However, the "as prepared" NCs, with their higher dopant concentration (and hence increased chance of exchange interaction between dopants), show a higher $M_{5 T} / M_{\text {sat }}$ than the "HCl-washed" samples, with their order of magnitude lower dopant concentration. Tsuji et al. posit that Mn ions on the surface do not interact magnetically. However, in this paper we show that even with uniform Heisenberg exchange constants for surface and interior atoms, taking into account the nonhomogeneous distribution of dopant atoms within the NC alone is sufficient to explain the magnetization results. 


\section{MODEL}

The experiments of Tsuji et al. are conducted with $3 \mathrm{~nm}$ diameter ZnS NCs $\stackrel{7}{\text { Using }}$ the zinc-blende model of Lippens and Lanoo, this corresponds to a 729 atom NC, with alternating "shells" of anion and cation atoms, summarized in Table $\coprod^{10}$ Assuming random incorporation of dopant atoms during the growth process, the distribution of dopant atoms within the crystals obeys a binomial law as noted by Counio et al. $\frac{1}{2}$ where the probability, $p\left(N_{d}\right)$, that a $\mathrm{NC}$ contains $N_{d}$ dopant atoms, is given by

$$
p\left(N_{d}\right)=\left(\begin{array}{c}
n_{\text {sites }} \\
N_{d}
\end{array}\right) x^{N_{d}}(1-x)^{n_{\text {sites }}-N_{d}}
$$

where

$$
\left(\begin{array}{l}
n \\
m
\end{array}\right)
$$

is a binomial coefficient, $x$ is the mean dopant concentration, and $n_{\text {sites }}$ is the number of cation sites into which the dopant atom may substitute itself. This is easily generalized to consider separate "inner" and "outer" volumes within the NC, consisting of $n_{\text {inner }}$ and $n_{\text {outer }}$ sites and having dopant concentrations $x_{\text {inner }}$ and $x_{\text {outer }}$, respectively. This is illustrated schematically in Figure 1

The magnetization ratio, $M_{5 T} / M_{\text {sat }}$ (using the notation of Tsuji et al.), is related to the observed susceptibility, $\chi_{o b s}$ and that expected of $N$ independent dopants of susceptibility $\chi_{1}$, and can be broken down into contributions of various dopant cluster geometries, $\stackrel{11,12}{12}$ by the expression

$$
\begin{aligned}
\left(M_{5 T} / M_{s a t}\right) & =\frac{\chi_{o b s} \mu_{0} B}{N_{d} \chi_{1} \mu_{0} B} \\
& =\sum_{i} P_{i} X_{i}
\end{aligned}
$$

where $i$ runs over the possible cluster types, $P_{i}$ is the probability of occurence of a given cluster of dopant atoms, and $X_{i}=\chi_{i} / \chi_{1}$ is the normalized susceptibility of cluster-type $i$, as calculated by Kreitman et $^{\text {al }}$. $^{12} X_{i}$ is a function of temperature ( $T=5 \mathrm{~K}$ in the experiment) and the nearest-neighbor Heisenberg exchange constant, which we take to be $-J / k_{b} \approx 10 \mathrm{~K}$ for $\mathrm{Mn}$ in bulk $\mathrm{ZnS}{ }^{13}$ With (anion-mediated) nearest-neighbor interactions, one obtains from a statistical mechanical treatment of the spin configurations as a function of $-k_{b} T / J$ the normalized cluster suceptibilities for all clusters up to three atoms as: $X_{\text {single }}=1.0$ (by definition), $X_{\text {pair }}=0.0, X_{\text {opentriangle }}=1.0$, and $X_{\text {closed triangle }}=0.1 .^{12}$ A more detailed description is given in the Appendix. The values of the $X_{i}$ are essentially constant for $5 \mathrm{~K} \leq-J / k_{b} \leq 50 \mathrm{~K}$, so our results are independent of the exact value of $J$. 
For the bulk case, the probabilities of occurence for the various clusters have been derived by Behringer 14 For nanocrystalline systems, this is unsatisfactory, due to the finite size and shape effects on the probability of forming various clusters; recently, Suyver et al. have performed numerical simulations and derived analytic results for the probability of dopant pairs in spherical NCs assuming homogeneous doping ${ }^{15}$ Less than $1 \%$ of Mn atoms are part of three-dopant clusters for the concentrations in our calculations, so the pair-only truncation would make a minor contribution to the magnetization ratio. However, we have included them in our calculations, as the determination is not particularly difficult. More important is the possibility of an inhomogeneous distribution of dopant atoms within the NC, illustrated schematically in Figure 11 We performed numerical simulations, in which Eq.(11) was used to determine a number of dopant atoms to be randomly placed within a given set of cation shells. $P_{i}$ was evaluated by counting all the types of (anion-mediated) nearest-neighbor clusters in a statistical sampling of $10^{6}$ dopant configurations. Then Eq. (3) was used to determine the magnetization ratio for the ensemble of doped NCs.

\section{RESULTS}

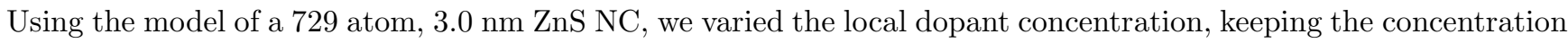
over the total NC equivalent to the magnetically determined concentration, $\bar{x}_{\text {curie }}$, from experiment. The shells are explicitly described in Table凹 and the results are shown in Table The higher average number of dopant atoms, $\bar{N}_{d}$, in our calculations reflects the fact that our model NC is slightly larger than that assumed by Tsuji et al.; otherwise we would expect $\bar{x}_{\text {curie }}$ to underestimate the number of dopants by a few percent. 13

We initially performed calculations in which the dopant atoms were uniformly distributed over all 420 cation sites; this results in a higher magnetization ratio for the low-concentration samples than for the high-concentration samples, contradicting the experimental results. However, we understand the acid washing procedure as removing any dopant atoms on the outermost shell of the NC. The $x_{N C}$ reported for the "HCl-washed" experimental samples corresponds to the number of dopant atoms in the interior divided by the total number of cation sites, $n_{\text {total }}$, in the $\mathrm{NC}$ (including the surface cation sites). But in the "HCl-washed" case, the remaining dopants are not in the outermost shell, but are constrained to be in the $n_{\text {inner }}$; we can determine $x_{\text {inner }}$ using the relation $x_{N C} n_{\text {total }}=x_{\text {inner }} n_{\text {inner }}$. Similar stoichiometric reasoning allows us to calculate $x_{\text {outer }}$. The calculations in Table II show the results of constraining the inner sites to consist of the one, two, three, and four cation shells, both with and without doping into the exterior (fifth) cation shell. For cases with an outer shell and higher $x_{N C}$ (modelling the "as prepared" experimental samples),

$x_{\text {outer }}>x_{\text {inner }}$ reduces the probability of pairing between surface and interior dopant atoms, increasing $M_{5 T} / M_{\text {sat }}$ as 
compared to the uniformly distributed case. However, the magnetization ratio for these high $x_{N C}$ samples is relatively insensitive to any restriction of the sites available for the interior dopant atoms, since the number of dopant atoms in this exterior shell is much higher than the number of dopant atoms in the interior, and to a first approximation is similar to the result obtaining by having all the dopant atoms on the exterior (since $x_{\text {outer }} n_{\text {outer }} \gg x_{\text {inner }} n_{\text {inner }}$ ).

Constraining the interior dopant atoms to reside within some locally concentrated sphere (for the purpose of our model, concentric with the NC), is qualitatively sufficient to achieve the proper effect of reducing the magnetization ratio for the washed samples with respect to the unwashed samples (as seen by comparing the respective rows of the "With Exterior Shell" and "Without Exterior Shell" calculations in Table 핑. In particular, constraining the interior dopant atoms to reside on the innermost cation shell nearly reproduces the experimental magnetization ratio results for both the "as prepared" (93.4\% theory, $91 \%$ experiment) and the "HCl-washed" ( $80.1 \%$ theory, $77 \%$ experiment), despite the simplicity of the model.

\section{CONCLUSION}

The inhomogeneous dopant distribution model reproduces the magnetization behavior observed by Tsuji et al.without assuming different magnetic interactions for surface and interior dopant atoms. As noted in Section III at the experimental temperature of $5 \mathrm{~K}$, Heisenberg exchange constants in the range of $5 \mathrm{~K} \leq-J / k_{b} \leq 50 \mathrm{~K}$ are indistinguishable 12 Further study on the effects of dopant geometry and NC size on $J$ may rely on electronic structure calculations, such as the recently developed ZILSH method of O'Brien and Davidson ${ }^{16}$

Our results suggests that dopant atoms in the interior of the $\mathrm{NC}$ are localized. It has been noted previously that $\mathrm{Mn}_{\mathrm{x}} \mathrm{Cd}_{1-\mathrm{x}} \mathrm{Se} \mathrm{NCs}$ appear to effectively zone-refine out the dopant atoms, since the energetic barrier to rearranging the lattice is not as insurmountable as in a bulk crystal $\underline{3}^{3}$ This suggest that there may exist a region near the center of the NC in which the energetic barrier is high enough to prevent migration of dopant atoms to the surface, surrounded by a region in which dopants migrate to the surface during synthesis and processing, as shown schematically in Figure 11 This may be further investigated by molecular dynamics simulations.

\section{ACKNOWLEDGEMENTS}

J.S. thanks the National Defense Science and Engineering Grant (NDSEG) program and U.S. Army Research Office Contract/Grant No. FDDAAD19-01-1-0612 for financial support. K.B.W. thanks the Miller Institute for Basic 
Research in Science for financial support. This work was also supported by the Defense Advanced Research Projects Agency (DARPA) and the Office of Naval Research under Grant No. FDN00014-01-1-0826.

\section{APPENDIX}

Below is a brief summary of the cluster susceptibility model, following Kreitman et al ${ }^{12}$ and Smart 11 . The Heisenberg Hamiltonian for nearest-neighbor interactions is

$$
\hat{H}=-2 J \sum_{n n(i, j)} \mathbf{S}_{i} \cdot \mathbf{S}_{j}+g \mu_{B} H_{0} \sum_{i} S_{i}^{z}
$$

where $J$ is the exchange constant, $n n(i, j)$ indicates that only nearest-neighbor $i$ and $j$ pairs are included, $\mathbf{S}_{i}$ is the spin-vector for site $i, g$ is the $g$-factor, $\mu_{B}$ is the Bohr magneton, $H_{0}$ is the applied field, and $S_{i}^{z}$ is the $z$ component of the spin on site $i$. There are three types of nearest-neighbor clusters containing three atoms or less: pairs $(D)$, closed triangles $(E)$ and open triangles $(N)$. The eigenenergies of Eq. (44) for these configuratations are respectively,

$$
\begin{aligned}
E_{D}= & -J[j(j+1)-2 S(S+1)]+g \mu_{B} H_{0} M \\
& 0 \leq j \leq 2 S,|M| \leq j \\
E_{E}= & -J[j(j+1)-2 S(S+1)]+g \mu_{B} H_{0} M \\
& 0 \leq k \leq 2 S,|k-S| \leq j \leq k+S,|M| \leq j \\
E_{N}= & -J[j(j+1)-k(k+1)-S(S+1)]+g \mu_{B} H_{0} M \\
& 0 \leq k \leq 2 S,|k-S| \leq j \leq k+S,|M| \leq j,
\end{aligned}
$$

using the standard notation of spin angular momentum. For $n \in\{D, E, N\}$, the free energy, $F$, is given by,

$$
-\beta F=\ln \sum_{n} e^{-\beta E_{n}}
$$

where $\beta=1 / k_{B} T, k_{B}$ is the Boltzmann constant and $T$ is the temperature. The susceptibility, $\chi$, is given by

$$
\begin{aligned}
\chi & =-\left.\left(\frac{\partial^{2} F}{\partial H_{0}^{2}}\right)\right|_{H_{0}=0} \\
& =-\left.\frac{\partial}{\partial H_{0}} \frac{g \mu_{B} \sum_{n}\left(\sum_{i} S_{i}^{z}\right)^{2} e^{-\beta E_{n}}}{\sum_{n} e^{-\beta E_{n}}}\right|_{H_{0}=0} \\
& =\left[\frac{\beta\left(g \mu_{B}\right)^{2} \sum_{n}\left(\sum_{i} S_{i}^{z}\right)^{2} e^{-\beta E_{n}}}{\sum_{n} e^{-\beta E_{n}}}-\left(\frac{g \mu_{B} \sum_{n}\left(\sum_{i} S_{i}^{z}\right)^{2} e^{-\beta E_{n}}}{\sum_{n} e^{-\beta E_{n}}}\right)^{2}\right]_{H_{0}=0} \\
& =\beta\left(g \mu_{B}\right)^{2}\left[\left\langle\left(\sum_{i} S_{i}^{z}\right)^{2}\right\rangle-\left\langle\sum_{i} S_{i}^{z}\right\rangle^{2}\right]_{H_{0}=0} .
\end{aligned}
$$


The susceptibility of a set of noninteracting $(J=0)$ spins, $\chi_{S}$, is simply,

$$
\chi_{S}=\frac{1}{3} \beta\left(g \mu_{B}\right)^{2} S(S+1) .
$$

We can then use this to definte a normalized susceptibility $X=\chi / \chi_{S}$, and likewise normalized susceptibilities of each cluster type $i$, as $X_{i}=\chi_{i} / \chi_{S}$, used in Eq.(3).

1 G. Counio, T. Gacoin, and J. P. Boilot, J. Phys. Chem. B 102, 5257 (1998).

${ }^{2}$ N. Feltin, L. Levy, D. Ingert, and M.-P. Pileni, Adv. Mater. 11, 398 (1999).

${ }^{3}$ F. V. Mikulec, M. Kunoa, M. Bennati, D. A. Hall, R. G. Griffin, and M. Bawendi, J. Am. Chem. Soc. 122, 2532 (2000).

${ }^{4}$ K. M. Hanif, R. W. Meulenberg, and G. F. Strouse, J. Am. Chem. Soc. 124, 11495 (2002).

${ }^{5}$ D. J. Norris, N. Yao, F. T. Charnock, and T. A. Kennedy, Nano Lett. 1, 3 (2001).

${ }^{6}$ H. Yang, P. H. Holloway, and B. B. Ratna, J. Appl. Phys. 93, 586 (2003).

7 N. Tsuji, H. Kitazawa, and G. Kido, J. Appl. Phys. 93, 6957 (2003).

8 J. Spalek, A. Lewicki, Z. Tarnawski, J. K. Furdyna, R. R. Galazka, and Z. Obuszko, Phys. Rev. B 33, 3407 (1986).

9 J. K. Furdyna, J. Appl. Phys. 64, R29 (1988).

10 P. E. Lippens and M. Lanoo, Phys. Rev. B 39, 10935 (1989).

11 J. S. Smart, in Magnetism, edited by G. T. Rado and H. Suhl (Academic Press, New York, 1963), vol. 3, pp. 63-114.

12 M. M. Kreitman, F. J. Milford, R. P. Kenan, and J. G. Daunt, Phys. Rev. 144, 367 (1966).

13 Y. Shapira, S. Foner, D. H. Ridgley, K. Dwight, and A. Wold, Phys. Rev. B 30, 4021 (1984).

14 R. E. Behringer, J. Chem. Phys. 29, 537 (1958).

15 J. F. Suyver, R. Meester, J. J. Kelly, and A. Meijerink, J. Lumin. 102-103, 182 (2003).

16 T. A. O'Brien and E. R. Davidson, Int. J. Quant. Chem. 92, 294 (2003). 


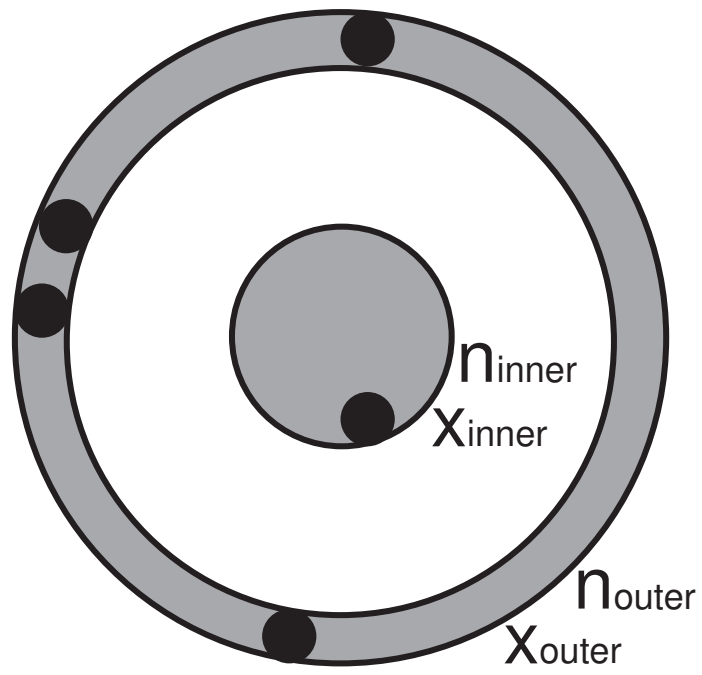

FIG. 1: Schematics of the inhomogeneous dopant distribution model proposed in this paper. $x_{\text {inner }}$ and $x_{\text {outer }}$ are the dopant concentrations for the inner and outer shells respectively. $n_{\text {inner }}$ and $n_{\text {outer }}$ are the number of cation sites enclosed within the inner and outer shells, respectively. Black circles show a random configuration of dopant atoms. For the low dopant concentration ("HCl washed") samples, the restriction of doping to a small inner shell increases the probability of pairs as compared to the case where the dopant atoms are uniformly distributed over the entire NC, hence reducing $M_{5 T} / M_{s a t}$. For high dopant concentration ("as prepared") samples, $x_{\text {outer }}>x_{\text {inner }}$ reduces the probability of pairing between surface and interior dopant atoms as compared with the uniformly distributed case, hence increasing $M_{5 T} / M_{\text {sat }}$.

\begin{tabular}{ccccc}
\hline Shell & Type & Atoms in Shell Total Cations Total Atoms \\
\hline 0 & Anion & 1 & 4 & 1 \\
1 & Cation & 4 & 5 \\
2 & Anion & 12 & & 17 \\
3 & Cation & 24 & 28 & 41 \\
4 & Anion & 42 & & 83 \\
5 & Cation & 64 & 92 & 147 \\
6 & Anion & 92 & & 239 \\
7 & Cation & 124 & 216 & 363 \\
8 & Anion & 162 & & 525 \\
9 & Cation & 204 & 420 & 729 \\
\hline
\end{tabular}

TABLE I: Description of the zinc-blende NC model of Lippens and Lanoc ${ }^{10}$ for a 3.0-nm diameter ZnS crystal. 


\begin{tabular}{|c|c|c|c|c|}
\hline$n_{\text {inner }}$ & $x_{\text {inner }} \% n_{\text {outer }}$ & $x_{\text {outer }} \% x_{N C} \%$ & $\bar{N}_{d}$ & $M_{5 T} / M_{s a t} \%$ \\
\hline
\end{tabular}

Uniformly Distributed

$\begin{array}{lllllll}420 & 1.20 & 0 & 0.00 & 1.20 & 5.03 & 90.3 \\ 420 & 0.10 & 0 & 0.00 & 0.10 & 0.42 & 99.1\end{array}$

With Exterior Shell

$\begin{array}{lllllll}216 & 0.19 & 204 & 2.27 & 1.20 & 5.02 & 93.7 \\ 92 & 0.46 & 204 & 2.27 & 1.19 & 5.01 & 94.5 \\ 28 & 1.50 & 204 & 2.27 & 1.19 & 5.00 & 94.0 \\ 4 & 10.48 & 204 & 2.27 & 1.17 & 4.91 & 93.4\end{array}$

Without Exterior Shell

$\begin{array}{ccccccc}216 & 0.19 & 204 & 0.00 & 0.10 & 0.42 & 98.2 \\ 92 & 0.46 & 204 & 0.00 & 0.10 & 0.41 & 96.2 \\ 28 & 1.50 & 204 & 0.00 & 0.10 & 0.40 & 90.6 \\ 4 & 10.48 & 204 & 0.00 & 0.07 & 0.31 & 80.1\end{array}$

Exterior Shell Only

$\begin{array}{lllllll}0 & 0.00 & 204 & 2.27 & 1.09 & 4.60 & 94.3\end{array}$

All Dopants on Exterior

\begin{tabular}{ccccccc}
0 & 0.00 & 204 & 2.47 & 1.20 & 5.02 & 93.8 \\
0 & 0.00 & 204 & 0.21 & 0.10 & 0.42 & 99.5 \\
\hline Experimental Results ${ }^{7}$ & & & & \\
& As prepared & & 1.2 & 4.3 & 91 \\
HCl-washed & & 0.10 & $0.3-0.5$ & 77 \\
\hline
\end{tabular}

TABLE II: Calculated results, for $10^{6} \mathrm{NC}$ sample. Definitions of the columns are given in Sections 2 and 3 . We found the values of $M_{5 T} / M_{\text {sat }}$ and $\bar{N}_{d}$ to vary only slighlty as compared to calculations using $10^{4}$ NCs. 